Relations industrielles

Industrial Relations

\title{
A l'échelle mondiale
}

\section{Lionel Moreau}

Volume 6, numéro 3, juin 1951

URI : https://id.erudit.org/iderudit/1023215ar

DOI : https://doi.org/10.7202/1023215ar

Aller au sommaire du numéro

Éditeur(s)

Département des relations industrielles de l’Université Laval

ISSN

0034-379X (imprimé)

1703-8138 (numérique)

Découvrir la revue

Citer cet article

Moreau, L. (1951). A l'échelle mondiale. Relations industrielles / Industrial Relations, 6(3), 86-89. https://doi.org/10.7202/1023215ar

Tous droits réservés @ C Département des relations industrielles de l’Université Laval, 1951
Ce document est protégé par la loi sur le droit d'auteur. L'utilisation des services d'Érudit (y compris la reproduction) est assujettie à sa politique d'utilisation que vous pouvez consulter en ligne.

https://apropos.erudit.org/fr/usagers/politique-dutilisation/ 
Les méthodes de recrutement, de sélection et de placement dans chacun des centres, ont joué un grand rôle dans toutes ces réalisations. La mise en application de ces méthodes a assuré le bon fonctionnement et l'efficacité des centres d'apprentissage.

Il est à souhaiter que par l'amélioration constante de telles méthodes, notre système d'apprentissage puisse toujours répondre aux besoins sans cesse croissants de notre industrie québecoise.

\section{A l'échelle mondiale}

\section{par LiONEL MOREAU, ptre}

Les problèmes de l'Economie politique, la production et la distribution des biens, ne peuvent plus se réduire à des problèmes de classe ou même à des problèmes nationaux. Ce sont des problèmes qui se posent aujourd'hui à l'échelle mondiale. Telle est l'affirmation principale que Sa Sainteté le Pape Pie XII faisait, le 3 juin dernier, au Congrès International d'études sociales et de l'Association internationale sociale chrétienne.

Après avoir ainsi situé le problème de l'équilibre à réaliser entre la production et la consommation des biens dans les cadres de l'économie mondiale, Sa Sainteté, poursuivant son enseignement de Summi Pontificatus et des allocutions de Noël sur la Paix, compte sur la solidarité universelle de la grande famille humaine et fait appel à la coalition des gens de bien : « arrière les préoccupations égoïstes de nationalités et de classes qui puissent gêner le moins du monde une action loyalement entreprise et vigoureusement menée dans la conspiration de toutes les forces et de toutes les possibilités sur toutes ies surfaces du globe, dans le concours de toutes les initiatives et de tous les efforts des individus et des groupes particuliers dans la collaboration universelle des peuples et des Etats, chacun apportant sa contribution respective de richesse : en matières premières, en capitaux, en main d'oeuvre».

De nos jours des études sérieuses sont entreprises par les économistes pour renflouer le système capitaliste. C'est que, note le T. R. Père Lévesque, sur le plan doctrinal, la majorité des individus, tant aux EtatsUnis qu'au Canada, continuent de croire au libéralisme et au système économique qui l'incarne. Mais leur foi a subi une évolution, car leur adhésion a cessé d'être totale pour devenir conditionnelle. Le capitalisme libéral n'est plus envisagé comme le seul régime possible et les phénomènes qu'il engendre, telles les crises, ne sont plus considérées comme inéluctables ${ }^{1}{ }^{1}$

Cette révolution sur le plan doctrinal est la conséquence logique d'une révolution sur le plan de la théorie économique. Mentionnons les principes exposés dans la Théorie générale de l'emploi, de l'intérêt et de la monnaie de Lord Kevnes, auxquels se refèrent le Fair Deal de Truman et le plan *semblable appliqué au Canada. De plus, le plan Marshall, les dons du Canada aux pays d'Europe, le plan Colombo proposé ces derniers temps par les Etats-Unis pour venir en aide à l'Asie, ont sans doute un but humanitaire des plus louables, mais s'inscrivent aussi dans ce contexte capitaliste.

Le système capitaliste se devait de se renouveler sous peine de disparaître. Il a tenté de promouvoir

(1) T.R.P. Georges-H. Lévesque, o.p., Réalisme économique et progrès social, Semaines Sociales de France, Lille, 1949, p. 90. 
le progrès social en élaborant son programme de stabilité économique. Le capitalisme passe par des phases diverses, au point que l'on se croirait en face de doctrines nouvelles avec le New Deal et le Fair Deal. Nous ne parlons pas évidemment du dirigisme qui s'est emparé de l'économie américaine depuis l'ouverture de la guerre de Corée.

Les statisticiens de l'O.N.U. ont fait une étude comparative de la richesse des divers pays pour 1949. Soixante-dix pays qui représentent $90 \%$ de la population mondiale et possèdent $95 \%$ des revenus du monde ont eu un revenu national de $\$ 515$ milliards. Les Etats-Unis à eux seuls ont eu $\$ 216$ milliards, ce qui donne un revenu par personne de $\$ 1,453$. Le Canada arrive deuxième, avec $\$ 870$ par personne. Les pays suivants sont: la Nouvelle-Zélande (856), la Suisse (849), la Suède (780), la Grande-Bretagne (773), le Danemark (689), l'Australie (679), la Norvège (587), la Belgique (582), etc. La Russie n'apparaît qu'au 23e rang avec un revenu de $\$ 308$ par personne. La Chine a un revenu moyen de \$27 par personne. Les pays qui n'ont pas un revenu moyen supérieur à $\$ 40$ par personne pour 1949 sont les suivants : Indonésie (25), Chine (27), Corée du Sud (35), Birmanie et Siam (36), Ethiopie et Libéria (38), Equateur, Haïti, Arabie et Yemen $(40)$.

Voici une idée de la puissance industrielle des Etats-Unis pour 1950. Ce pays fabriquait l'an dernier, par mois, 500,000 réfrigérateurs, 450.000 machines à laver, 950,000 radios, 550,000 télévisions, 4 millions de complets d'hommes, 52 millions de paires de bas de nylon, 700,000 voitures et 110,000 camions. Il employait 13 ou 14 millions de plus de travailleurs qu'en 1939 et produisait 2 fois plus d'acier qu'au début de la dernière grande guerre.

Malgré que le niveau de vie soit le plus élevé du monde dans les pays où la grande corporation est la plus répandue, nous devons dire que le capitalisme, en regard des normes idéales du progrès social, est loin d'avoir produit ses fruits les plus parfaits.

La prospérité économique et le progrès social exigent dans un pays industrialisé que le produit national soit stable et maximum, qu'il soit réparti équitablement entre tous les facteurs de productition de ce revenu national et que l'utilisation optima des revenus des individus soit garantie.

Or, un auteur (J.-A. Estey) dit que de 1796 à 1920 les Etats-Unis ont connu 14 cycles d'une durée moyenne de 9 ans, et que le rapport entre les années de prospérité et de dépression a été de 1.6 à 1 . Un autre (Moulton) dit que les ressources productives des Etats-Unis n'ont pas été utilisées à plus de $80 \%$ de 1925 à 1929. Les enquêtes publiques sur les trusts et les cartels révèlent qu'il existe une distribution injuste des richesses ou du revenu national entre les divers facteurs de production. Enfin, la publicité qui devrait informer les consommateurs sur les qualités des produits mis en vente pour satisfaire leurs besoins, forme leur goût dans un sens préjudiciable, crée chez eux de nouveaux besoins pour des produits moins utiles sinon parfaitement nuisibles, mais qu'il est plus facile de produire en série. $\mathrm{Au}$ bilan, les classes qui ont la part la moins importante du revenu national ne l'utilisent pas de manière à en retirer le maximum de bien-être. ${ }^{2}$

En face de ces problèmes, les pays capitalistes se sont tournés de plus en plus vers une économie libérale mitigée. Le programme du New Deal est une tentative d'économie dirigée à base de moyens d'ordre monétaire. Mais nous savons aujourd'hui que si les moyens d'ordre monétaire sont absolument nécessaires à la reprise des affaires, à la' suite d'une période de dépression, ils ne

(2) Résumé de l'argumentation du R.P. Lévesque dans le cours cité plus haut. 
peuvent cependant à eux seuls provoquer la prospérité. Il faut qu'il y ait un certain équilibre entre les < placements > et les «dépenses de consommation .

L'Etat, disent gouvernants et capitalistes modernes, doit intervenir pour maintenir cet équilibre. En période inflationnaire, son rôle est de retirer de forts montants d'argent par taxation; en période de crise, c'est de diminuer de beaucoup les taxes, et par ailleurs d'ouvrir de vastes chantiers pour augmenter les - placements > et enrayer le chômage. L'Etat doit voir encore par sa taxation et sa sécurité sociale à mieux redistribuer le revenu national entre les différentes classes de la société. Il y arrivera par l'impôt progressif d'un côté et les mesures d'assistance et d'assurance sociales de l'autre.

Puisque les crises économiques viennent à la suite de variations économiques trop fortes et trop brusques, l'Etat aura donc le devoir de compenser ces fluctuations venant $d u$ secteur privé de l'économie nationale par l'aménagement de son propre budget. La sagesse de ses gouvernants se manifestera par le choix judicieux du moment le plus efficace d'intervention de la manière rationnelle que nous venons de décrire.

Le capitalisme a-t-il trouvé sa voie? Est-il capable d'appliquer sans compromis le principe thomiste de la destinée sociale des biens possédés privément? Est-il capable d'enrayer les crises cycliques? Nous ne le croyons pas. Et d'abord nous répondons en faisant appel aux faits. Dès l'automne de 1949, les signes évidents d'une crise sont apparus aux Etats-Unis et au Canada. Tout au long de l'hiver, le nombre des chômeurs n'a fait que s'accroître. Nous serions sous les effets de cette crise si de nouveau la production de paix n'avait pas été orientée vers la production de guerre à partir de 1950 , afin de répondre aux besoins de la guerre de Corée et du réarmement de l'Europe occidentale ainsi que des pays de l'Amérique du Nord.

De plus ces tentatives de réformes sont trop inspirées par des préoccupations capitalistes et nationales pour qu'elles puissent apporter le remède à la crise actuelle du monde. * L'on n'a que trop fait l'essai de la production en masse, d'exploitation jusqu'à l'épuisement de toutes les ressources du sol et du sous-sol, en sacrifiant durement à ces essais la population et l'économie paysannes ", comme le dit Sa Sainteté dans son discours du 3. juin 1950. M. Pierre Bayart ${ }^{3}$, qui analyse ce discours de S. S. Pie XII, continue ainsi l'argumentation: \&a vraie richesse d'un peuple ne se mesure pas à des critères purement quantitatifs, mais à la structure sociale d'une communauté dont la Famille est la base ».

Ces tentatives de réformes sont encore trop égoïstes. Elles oublient ie renouvellement nécessaire et l'épanouissement de l'esprit de justice, d'amour et de paix.

Nous pourrions illustrer toutes ces affirmations. Nous pourrions ajouter d'autres raisons à l'effet de démontrer que le capitalisme se doit de se renouveller sous peine de disparaître, mais dans un sens tout autre que celui dans lequel il s'est engagé par instinct de conservation. Cette orientation nouvelle que doit prendre l'économie mondiale a été clairement exposée au monde par Sa Sainteté Pie XII dans son discours du 3 juin 1950.

M. Bayart note avec justesse que ce Discours du Très Saint Père ne traite pas uniquement du problème de la co-gestion dans l'entreprise, mais d'abord et avant tout du problème du chômage qui dans son imminente et permanente menace

(3) Réorganisation de l'Economie mondiale et Co-gestion dans l'Entreprise, analyse du Discours de S.S. Pie XII du 3 juin 1950 au Congrès international d'études sociales et de l'Association Internationale Sociale Chrétienne, par Pierre Bayart, professeur à la Faculté libre de Droit de Lille. 
- risque d'affamer des milliers d'hommes, toute l'immense multitude des malheureux .... * Ce problème du chômage périodique, dit M. Bayart, c'est le problème crucial du capitalisme, et plus exactement d'un régime économique fondé sur le développement dynamique du progrès technique qui, par le mouvement qu'il imprime à l'activité économique, engendre nécessairement, dans sa marche en avant, des variations, les fluctuations de la conjoncture et des crises, cycliques ou non ». Voici le résumé de la Pensée pontificale dans ce discours du 3 juin, tel que présenté par M. Bayart :

Le rejet des \& mécanismes d'organisations sociales en contradiction avec l'ordre établi par Dieu », c'està-dire de la planification intégrale du système soviétique;

le rejet d'un retour pur et simple a un libéralisme condamné par l'Eglise et dépassé par les faits, qui se fierait, pour rétablir une économie normale, au seul mécanisme des lois du marché ;

la mise en garde contre une déformation de la sécurité sociale par une conception socialisante qui \& chargerait un Etat-Providence du soin de procurer à chacun de ses ressortissants, et pour toutes les circonstances de la vie, le droit à des exigences au bout du compte irréalisables »;

la mise en garde contre certaines conceptions de plein emploi réalisé par des formalismes trop artificiels; la nécessité primordiale, pour réaliser cette politique du plein emploi, et la première des sécurités sociales: celle du travail, d'organiser, à la source même de l'économie, et en évitant les excès d'un productivisme sans frein, une \& productivité saine et normale, sagement mesurée aux besoins et à la dignité de l'homme , et ordonnée autour de cette unité organique, constitutive de la' Société, qu'est la Famille ;

le maintien, à la base de l'économie, de l'entreprise privée, avec toutes les amodiations correspondant à son caractère social, mais en maintenant néanmoins son caractère privé et en laissant au chef d'entreprise sa responsabilité personnelle et la maîtrise de ses décisions économiques, et en sauvegardant autant que possible, contre le double danger du capitalisme anonyme et de la socialisation, cette portion la plus saine de l'économie que sont les exploitations individuelles ou familiales ;

l'affirmation, une fois de plus réitérée, de la nécessité primordiale d'une organisation professionnelle.

Tel est le sens de l'orientation nouvelle que le Très Saint Père indique à l'économie mondiale. Il invite maintenant théoriciens et techniciens « à concentrer leur attention et à faire converger leurs études . . . sur l'imminente et permanente menace du chômage, le plus imnortant, le plus urgent problème, celui qui pèse comme un cauchemar, précisément sur ces vieux pays d'industrie. . .»

\section{Vers une union des familles}

Les 23 et 24 avril dernier, le Département des relations industrielles de la Faculté des sciences sociales de Laval accueillait, dans la vaste salle de spectacles du Château Frontenac, près de cinq cents délégués venus de tous les coins de la province et des milieux les plus divers: patrons, ouvriers, directeurs de personnel, con- tremaîtres, officiers d'associations patronales et ouvrières, représentants de comités paritaires, aviseurs moraux, fonctionnaires du travail, représentants de groupements sociaux, etc.

Deux jours durant, les congressistes ont considéré, dans la sérénité qui convient et sous la direction éclairée de savants conférenciers et 